\section{Endothelial barrier antigen- immunoreactivity is conversely associated with blood-brain barrier dysfunction after embolic stroke in rats}

\author{
J. Pelz, ${ }^{1}$ W. Härtig, ${ }^{2}$ C. Weise, ${ }^{1}$ \\ C. Hobohm, ${ }^{1}$ D. Schneider, ${ }^{1}$ M. Krueger, ${ }^{3}$ \\ J. Kacza, ${ }^{4}$ D. Michalski ${ }^{1}$
}

'Department of Neurology, University of Leipzig

2Paul Flechsig Institute for Brain

Research, University of Leipzig

IInstitute of Anatomy, University of

Leipzig

${ }^{4}$ Institute of Anatomy, Histology and

Embryology, Faculty of Veterinary

Medicine, University of Leipzig, Germany

\section{Abstract}

While the concept of the Neurovascular Unit (NVU) is increasingly considered for exploring mechanisms of tissue damage in ischemic stroke, immunohistochemical analyses are of interest to specifically visualize constituents like the endothelium. Changes in immunoreactivity have also been discussed to reflect functional aspects, e.g., the integrity of the bloodbrain barrier (BBB). This study aimed to characterize the endothelial barrier antigen (EBA) as addressed by the antibody SMI-71 in a rat model of embolic stroke, considering FITC-albumin as BBB leakage marker and serum levels of BBB-associated matrix metalloproteinases (MMPs) to explore its functional significance. Five and $25 \mathrm{~h}$ after ischemia onset, regions with decreased BBB integrity exhibited a reduction in number and area of EBA-immunopositive vessels, while the stained area per vessel was not affected. Surprisingly, EBA content of remaining vessels tended to be increased in areas of BBB dysfunction. Analyses addressing this interrelation resulted in a significant and inverse correlation between the vessels' EBA content and degree of BBB permeability. In conclusion, these data provide evidence for a functional relationship between EBA-immunoreactivity and BBB dysfunction in experimental ischemic stroke. Further studies are required to explore the underlying mechanisms of altered EBA-immunoreactivity, which might help to identify novel neuroprotective strategies.

\section{Introduction}

As consequence of numerous neuroprotective failures in acute ischemic stroke, ${ }^{1}$ the perspective of tissue damage shifted from the initial neurocentric to an extended view considering the vasculature as an essential component of the so-called Neurovascular Unit (NVU). ${ }^{2-4}$ The NVU including endothelial and associated cells like neurons and glia also forms the bloodbrain barrier (BBB),, 5 which critically impacts on local homeostasis and ischemia-related complications like edema generation and hemorrhagic transformation. ${ }^{8}$ However, to gain further insight into the functional role of NVU constituents, their reliable visualization under experimental conditions is required and largely based on specific markers allowing the simultaneous detection of multiple vascular compartments. In this context, antibodies directed against the endothelial barrier antigen (EBA) have frequently been applied to address the cerebral endothelium in rodents also under pathological conditions like ischemic stroke, ${ }^{9-12}$ intracerebral hemorrhage, ${ }^{13}$ experimental allergic encephalomyelitis, ${ }^{14}$ traumatic brain injury, ${ }^{15}$ and spinal cord trauma. ${ }^{16}$ Initially described in 1987 by Sternberger and Sternberger, ${ }^{17}$ anti-EBA is a monoclonal mouse antibody of the immunoglobulin M ( $(\mathrm{ggM})$ class, that binds to the luminal side of cerebral endothelial cells. The name EBA was chosen due to the assumption that antigen reactivity is associated with parts of the cerebral vasculature exhibiting a selective permeability barrier, since EBA-immunoreactivity in the rat brain was found to be absent or diminished in regions with naturally restricted BBB integrity like the area postrema and the choroid plexus. ${ }^{17}$ Subsequently, EBA was also detected in endothelial cells in the reproductive tract of male rats, probably as a part of the blood-testis barrier. ${ }^{18}$ However, the distribution of EBA varies strongly in the CNS with a mixture of EBA-immunopositive and -immunonegative cells in pial vessels,$^{19} \mathrm{a}$ complete lack of EBA in cerebral arterioles and a mosaic pattern in capillaries and venules. ${ }^{20}$ Notably, previous studies demonstrated that cerebral areas with reduced EBA-positive vessels provide increased BBBpermeability for endogenous albumin and IgG, ${ }^{13,21,22}$ leading to the hypothesis that EBAimmunoreactivity might represent a potential tool to explore functional characteristics of the NVU with a special focus on BBB integrity. However, this perspective has so far not been investigated under ischemic conditions, although such a feature might notably complement further research while covering ischemiarelated consequences in more detail.

The present study therefore aimed to charac-
Correspondence: Dr. Dominik Michalski, University of Leipzig, Department of Neurology, Liebigstr. 20, 04103 Leipzig, Germany.

Tel. +49.341.9724206 - Fax: +49.341.9724199.

E-mail: dominik.michalski@medizin.uni-leipzig.de

Keywords: ischemic stroke, embolic model, EBA, blood-brain barrier, matrix metalloproteinase.

Acknowledgments: the authors would like to thank Dr. Johannes Boltze (Fraunhofer Institute for Cell Therapy and Immunology, Leipzig, and Translational Centre for Regenerative Medicine, University of Leipzig, Germany) for assistance in planning the animal experiments. Further, Dr. Fink Madaj-Sterba and Ms. Sigrid Weisheit (Medizinisch-Experimentelles Zentrum, University of Leipzig) are acknowledged for animal care, Dr. Manja Kamprad (Institute of Clinical Immunology and Transfusion Medicine, University of Leipzig) for initial quantification on MMP serum levels, and Ms. Ute Bauer (Paul Flechsig Institute for Brain Research, University of Leipzig) for supporting tissue preparation.

Contributions: DM, $\mathrm{CH}$, WH, conceived the underlying study setup; DS, facilitated funding; DM, CW, conducted animal experiments; JP, WH, performed tissue preparation and serial staining; JP, JK, developed strategy for quantitative analyses; MK, performed additional imaging analyses; JP, analyzed the data; JP, DM, wrote the manuscript; WH, JK, manuscript critical revisions.

Conflict of interests: the authors declare no conflict of interests.

Received for publication: 25 May 2013. Accepted for publication: 18 October 2013.

This work is licensed under a Creative Commons Attribution NonCommercial 3.0 License (CC BYNC 3.0).

(1)Copyright J. Pelz et al., 2013

Licensee PAGEPress, Italy

European Journal of Histochemistry 2013; 57:e38 doi:10.4081/ejh.2013.e38

terize EBA in a descriptive and functional fashion using an embolic model of focal cerebral ischemia in rats. For this purpose, EBAimmunoreactivity was analyzed to identify the spatio-temporal pattern in ischemia-affected areas exhibiting increased BBB permeability as identified by leakage of intravenously applied fluorescein isothiocyanate (FITC)-albumin. Moreover, relationships between different aspects of EBA-immunoreactivity and BBB permeability as well as matrix metalloproteinases (MMPs), known to promote disintegration of the BBB during stroke, ${ }^{8,23}$ were analyzed by correlation coefficients to further explore the functional significance of EBA under ischemic conditions. 


\section{Materials and Methods}

\section{Study design and tissue preparation}

Seventeen male Wistar rats (mean body weight $324 \mathrm{~g}$ ), provided by Charles River (Sulzfeld, Germany), underwent embolic middle cerebral artery occlusion as described below and were consecutively assigned to an observation period of 5 or $25 \mathrm{~h}$, respectively. Premature death occurred in one animal, resulting in a total of 16 animals ( 8 in each group) that finished the study addressing spatio-temporal characteristics of EBA-immunoreactivity. In control experiments, 4 rats (mean body weight $358 \mathrm{~g}$ ) underwent sham-operation, while additional 2 rats suffering from embolic middle cerebral artery occlusion (mean body weight $322 \mathrm{~g}$ ) were used for triple fluorescence labeling of vascular constituents. For visualizing ischemia-associated changes in BBB integrity, 4 or $24 \mathrm{~h}$ after ischemia onset FITC-albumin (molecular weight about $70 \mathrm{kDa}$; $20 \mathrm{mg}$ dissolved in $1 \mathrm{~mL}$ saline; Sigma, Taufkirchen, Germany), usually not crossing the BBB under physiological conditions, ${ }^{24}$ was applied intravenously via a femoral catheter. After an additional 1-hour circulation period animals were sacrificed and blood was sampled for measuring serum levels of MMPs, followed by transcardial perfusion with a fixative containing $4 \%$ paraformaldehyde in phosphate-buffered saline (PBS). Next, brains were removed from the skulls and immersed in the same fixative for 24 hours before their equilibration in $30 \%$ phosphate-buffered sucrose. Free-floating $30 \mu \mathrm{m}$ thick coronal sections from the entire forebrain, stored in $0.1 \mathrm{M}$ Tris-buffered saline, pH 7.4 (TBS), containing sodium azide, served for subsequent histological analyses. The experimental protocol involving animals was approved by local authorities (Regierungspräsidium Leipzig, reference numbers TV-02/09 and TVV-34/11). Care and treatment of animals conformed to the standards of the European Communities Council Directive (86/609/EEC).

\section{Experimental induction of focal cerebral ischemia and sham procedure}

Focal cerebral ischemia was induced by an embolic model originally described by Zhang $e t$ $a l .{ }^{25}$ with some minor modifications as previously reported. ${ }^{24}$ Briefly, animals were anaesthetized breathing 2.0-2.5\% isoflurane in a mixture of $70 \% \mathrm{~N}_{2} \mathrm{O}$ and $30 \% \mathrm{O}_{2}$. During the surgical procedure the body temperature was adjusted to $37^{\circ} \mathrm{C}$ using a warming pad with a rectal probe (Fine Science Tools, Heidelberg, Germany). After preparation of left femoral vessels and catheter insertion, a tissue-conserving preparation of right cervical vessels was performed. Afterwards, a pointed PE-50 catheter was inserted into the right external carotid artery and advanced into the distal internal carotid artery where a blood clot with a length of approximately $45 \mathrm{~mm}$ was released. Finally, the catheter was removed and the stump of the external carotid artery ligated. The sham-procedure was performed in analogous manner, except of catheter insertion into cervical vessels and thus without clot release.

\section{Immunofluorescence labeling of EBA and enhancement of FITC-albumin}

A first series of brain sections was washed 3 times for 10 min with TBS before being rinsed with distilled water, mounted on fluorescencefree glass slides, air-dried and coverslipped with Entellan in toluene (Merck, Darmstadt, Germany). Afterwards, sections were screened using a fluorescence microscope (Axioplan; Zeiss, Germany) to identify regions with increased BBB permeability indicated by green fluorescence of extravasated FITC-albumin. Preceding brain sections were then applied to indirect red fluorescent Cy (carbocyanine) 3 staining of EBA-immunoreactivity and concomitant enhancement of FITC-albumin fluorescence. In detail, brain sections were washed 3 times for 10 min each with TBS before being blocked for 1 hour with TBS containing 5\% normal donkey serum and $0.3 \%$ Triton X-100 (TBSNDS-T) followed by an overnight incubation with SMI-71, an IgM mouse monoclonal antibody directed against EBA (1:400 in TBS-NDS-T; Covance, Emeryville, CA, USA). After further 3 washings with TBS for 10 min each, a cocktail was added for $1 \mathrm{~h}$ containing Cy2-anti-FITC-IgG (20 $\mu \mathrm{g} / \mathrm{mL}$ TBS-BSA; Dianova, Hamburg, Germany), for enhancement and long-term stabilization of the green fluorescent signal of FITC-albumin, and Cy3-donkey-anti-mouse-IgM ( $20 \mu \mathrm{g} / \mathrm{mL}$ TBS-BSA; Dianova) visualizing SMI71.

In additional experiments, selected brain sections from animals that underwent middle cerebral artery occlusion and injection of FITCalbumin were blocked with TBS-NDS-T for $1 \mathrm{~h}$ and then incubated overnight with a mixture of two endothelial markers, biotinylated Solanum tuberosum agglutinin $(20 \mu \mathrm{g} / \mathrm{mL}$ TBS-NDS-T; Vector, Burlingame, CA, USA) and SMI-71 (1:200). Next, the sections were rinsed with TBS and processed with a cocktail composed of Cy5-streptavidin (Dianova), Cy2-antiFITC-IgG and Cy3-donkey-anti-mouse-IgM (all at $20 \mu \mathrm{g} / \mathrm{mL}$ TBS-BSA) for $1 \mathrm{~h}$. Finally, brain sections were washed, mounted, air-dried and coverslipped as described above.

\section{Quantification of EBA-immunore- activity}

Fluorescence-based measurements were done by a blinded investigator using an Axioplan 2 microscope (Zeiss) with a structured-light confocal system (OptiGrid; Qioptiq, Fairport, NY, USA) and a digital camera (ORCAER; Hamamatsu, Hamamatsu City, Japan). Applying a 10x magnification, brain sections were first scanned for regions with clear extravascular green fluorescence signal in terms of FITC-albumin leakage arising from the inner part of the vessels indicating increased BBB permeability. Within those regions, 2 microscopic fields (each covering an area of about $0.55 \mathrm{~mm}^{2}$ ) were digitized and the total number as well as the stained area of EBAimmunopositive vessels vessels were assessed automatically using the imaging software Volocity 4.3.2 (PerkinElmer, Waltham, MA, USA). Criteria to identify a vessel were a length of at least $12 \mu \mathrm{m}$ and an area of at least $100 \mu^{2}$. Thereby, the following settings were applied: Find objects by intensity and exclude objects by size $\left(<100 \mu^{2}\right)$, while the overall technical standard was a 10x magnification with numerical aperture 0.3. Further settings were: Immersion medium air, exposure time $400 \mathrm{~ms}$, gain 0 , offset 0 , binning 1 , grid wide field, Cy2: excitation $480 \mathrm{~nm}$, emission $527 \mathrm{~nm}$, Cy3: excitation $545 \mathrm{~nm}$ and emission $610 \mathrm{~nm}$. The stained area per vessel was obtained by dividing the area of vessels by their total number. To obtain intensity of the EBA-immunofluorescence signal per $\mu^{2}$ vessel area, representing the vessels' EBA content, the total fluorescence intensity of the microscopic field was measured by Volocity 4.3.2 and manually corrected for the surprisingly low background staining. Finally, the background-corrected intensity of the EBAimmunofluorescence signal was divided by the previously obtained vessel area. For control issues on the ischemia-affected hemisphere, number and area of EBA-immunopositive vessels, area per vessel and the intensity of the EBA-immunofluorescence signal per $\mu \mathrm{m}^{2}$ vessel area were also measured in the hippocampus. Due to the nature of embolic stroke with varying infarct degrees and locations between animals ${ }^{26}$ we also analyzed corresponding regions of the contralateral (non-ischemic) hemisphere, allowing the calculation of a ratio between the ischemic and non-ischemic hemispheres for vessels' EBA content, finally enabling intergroup comparison. In sections from sham-processed animals devoid of FITCalbumin leakage, 4 microscopic fields in both hemispheres (each $0.55 \mathrm{~mm}^{2}$ ), covering hypothalamus, striatum, parietal cortex and hippocampus, were analyzed as described above. For illustration of data, high-resolution images were taken from selected sections using the confocal laser-scanning microscopes 510 
(Meta) or 700 (both from Zeiss) as well as an Olympus BX51 microscope equipped with an XM10 digital camera (Olympus, Hamburg, Germany). In addition, a coronal brain section was scanned using a photo scanner (2.000 dpi; ScanMaker 9800 XL; Microtek, Willich, Germany). Original images were generally processed with CorelDraw/ Photo-Paint version 12.0 (Corel Corp., Ottawa, Canada); if indicated, brightness, contrast or intensity were slightly adjusted without creating or deleting signals.

\section{Quantification of BBB integrity and measurement of BBB-related serum markers}

Quantification of BBB permeability in ischemia-affected brain regions using FITCalbumin as leakage marker as well as acquisition of MMP-2 and MMP-9 serum levels were described previously. ${ }^{27}$ The resulting ratio of extra-/intravascular Cy2-enhanced FITC-albumin as surrogate for BBB integrity as well as MMP serum levels were utilized for calculations in the present study.

\section{Statistical analysis}

Calculations were performed with SPSS version 20.0 (IBM Corp.; New York, NY, USA). The Wilcoxon test was utilized to check for statistical significance between values of the ischemic $v s$ the non-ischemic hemisphere at a single time point. For inter-group comparisons at different time points the $t$-test was used. To explore interrelations between assessed variables, partial correlations, controlled for observation time, were applied. In general, a $\mathrm{P}<0.05$ was considered as statistically significant.

\section{Results}

\section{EBA-immunoreactivity after exper- imental ischemic stroke}

In sham-processed animals devoid of focal cerebral ischemia, anti-EBA reliably detected cerebral vessels in neocortical and subcortical regions except of the circumventricular organs, e.g., the choroid plexus and some pial vessels showing an incomplete staining. Compared to the neocortex and striatum, the number of EBA-immunopositive vessels appeared to be symmetrically reduced in the hippocampus.

To ensure the specificity of endothelial EBAimmunolabeling in ischemia-affected brain regions, triple fluorescence labeling was applied resulting in nearly complete congruence of EBA-immunoreactivity and the endothelium as visualized by Solanum tuberosum lectin (STL), confirmed by FITC-albumin that remained intravascularly (Figure 1A). In contrast to the surrounding tissue with regular BBB integrity and to the non-affected contralateral hemisphere, vessel-associated EBAimmunoreactivity was found to be rarefied in areas with ischemia-related BBB dysfunction, indicated by FITC-albumin leakage (Figure 1B). Apart from the striatum as primary region of ischemia, this feature was also seen in the neocortex if affected by ischemia. A remarkably weak background staining in all animals, facilitated quantitative analyses of the red fluorescence signal.

\section{Diverse aspects of EBA-immunore- activity in regions with decreased \\ BBB integrity}

Sham-proceeded animals showed no differences in number of EBA-immunopositive vessels, stained area of vessels and area per vessel between both hemispheres (data not shown). Compared to corresponding regions in the non-affected hemisphere, $5 \mathrm{~h}$ after ischemia onset the number of EBAimmunopositive vessels was significantly reduced in the area of decreased BBB integrity as indicated by FITC-albumin leakage (Wilcoxon, $\mathrm{P}=0.02$; overview in Table 1), while stained vessel area provided the same tendency, scarcely missing statistical significance (Wilcoxon, $\mathrm{P}=0.09$ ). Similar findings were obtained at $25 \mathrm{~h}$ after ischemia onset with a significant decrease in the stained area of ves- sels (Wilcoxon, $\mathrm{P}=0.04$ ) and a reduction in number of EBA-immunopositive vessels (Wilcoxon, $\mathrm{P}=0.05$ ), simultaneously occurring with FITC-albumin leakage (Figure 2). Surprisingly, the stained area per vessel did not change at both time points (Wilcoxon, $5 \mathrm{~h}$ : $\mathrm{P}=0.12 ; 25$ h: $\mathrm{P}=0.35$ ).

Additionally, at both time points no significant changes in number of EBA-immunopositive vessels, stained vessel area or area per vessel were found between the ipsi- and contralateral hippocampus (Wilcoxon, $\mathrm{P}>0.05$ each).

\section{Vessels' EBA content in regions with BBB dysfunction}

Five and $25 \mathrm{~h}$ after ischemia onset, intensity of EBA-immunofluorescence signal per $\mu \mathrm{m}^{2}$ vessel area as surrogate for vessels' EBA content tended to be increased in areas with FITCalbumin leakage compared to corresponding regions on the non-affected hemisphere, however, failing statistical significance (Wilcoxon, $\mathrm{P}=0.12$ and $\mathrm{P}=0.17$ ). Even clearer in the hippocampus, no significant change in vessels EBA content was found at both time points (Wilcoxon, $\mathrm{P}>0.05$ each). Concerning the general time course, regions with decreased BBB integrity exhibited a slightly increased EBAimmunofluorescence signal per $\mu^{2}$ vessel area towards the 25 h time point, which, however, failed statistically significance when compared to $5 \mathrm{~h}(t$-test, $\mathrm{P}=0.58)$.

\section{Interrelations among number of EBA-immunopositive vessels, stained vessel area, vessels' EBA content and degree of BBB dys- function}

As previously described, in the applied model of focal cerebral ischemia affected brain regions displayed a decreased tendency of the extra-/intravascular FITC-albumin ratio as surrogate for BBB dysfunction between 5 and $25 \mathrm{~h}$ after ischemia onset, indicating an increase in BBB integrity in the early phase of stroke..$^{27}$ However, in areas with increased BBB perme-

Table 1. Parameters of endothelial barrier antigen (EBA)-immunoreactivity in the rat brain affected by focal cerebral ischemia.

\begin{tabular}{|c|c|c|c|c|c|c|c|c|}
\hline & EBA- & $\begin{array}{l}\text { r of } \\
\text { 10positive }\end{array}$ & Staine & & Are & & $\begin{array}{r}\text { In } \\
\text { EBA-in } \\
\mu m^{2}\end{array}$ & $\mathrm{al} /$ \\
\hline & $5 \mathrm{~h}$ & $25 \mathrm{~h}$ & $5 \mathrm{~h}$ & $25 \mathrm{~h}$ & 5 h & $25 \mathrm{~h}$ & $5 \mathrm{~h}$ & $25 \mathrm{~h}$ \\
\hline & $\downarrow$ & $\downarrow$ & $\downarrow$ & $\downarrow$ & & & $\uparrow$ & $\uparrow$ \\
\hline Area with BBB dysfunction & $(0.02)$ & $(0.05)$ & $(0.09)$ & $(0.04)$ & $=$ & $=$ & $(0.12)$ & $(0.17)$ \\
\hline Hippocampus & $=$ & $=$ & $=$ & $=$ & $=$ & $=$ & $=$ & $=$ \\
\hline
\end{tabular}

EBA, endothelial barrier antigen; BBB, blood-brain barrier. Arrows indicate an increased $(\uparrow)$, decreased $(\downarrow)$, or equal $(=)$ reaction of listed parameters in regions of ischemia-related BBB dysfunction compared to the contralateral (non-affected) hemisphere. P-values originating from the Wilcoxon test are shown in brackets. 
ability no significant correlation (corrected for time points) was found between the number or area of EBA-immunopositive vessels and the ratio of extra-/intravascular FITC-albumin $(\mathrm{r}=0.22, \mathrm{P}=0.47 ; \mathrm{r}=0.05, \mathrm{P}=0.87)$.

When further corrected for serum levels of MMP-2 and -9, the ratio of vessels' EBA content and the intensity of EBA-immunofluorescence signal per $\mu^{2}$ vessel area correlated in an inverse manner with the ratio of extra-/intravascular FITC-albumin in regions of increased BBB permeability $(\mathrm{r}=-0.68, \mathrm{P}=0.02 ; \mathrm{r}=0.60 \mathrm{P}=0.05$; Figure 3). No correlation was found between intensity of either EBA-immunosignal per $\mu^{2}$ vessel area or ratio of vessels' EBA content and serum levels of both MMP-2 and -9 (MMP-2: $\mathrm{r}=$ $-0.03, \mathrm{P}=0.91 ; \mathrm{r}=-0.2, \mathrm{P}=0.52 ; \mathrm{MMP}-9$ : $\mathrm{r}=$ $-0.02, \mathrm{P}=0.95 ; \mathrm{r}=-0.11, \mathrm{P}=0.72)$.

\section{Discussion}

Following previous investigations, regular establishment of EBA has been considered to be associated with an intact cerebral vasculature as well as unaltered BBB integrity, ${ }^{13,17,21,22}$ although clear evidence for such a functional perspective is still missing, especially under
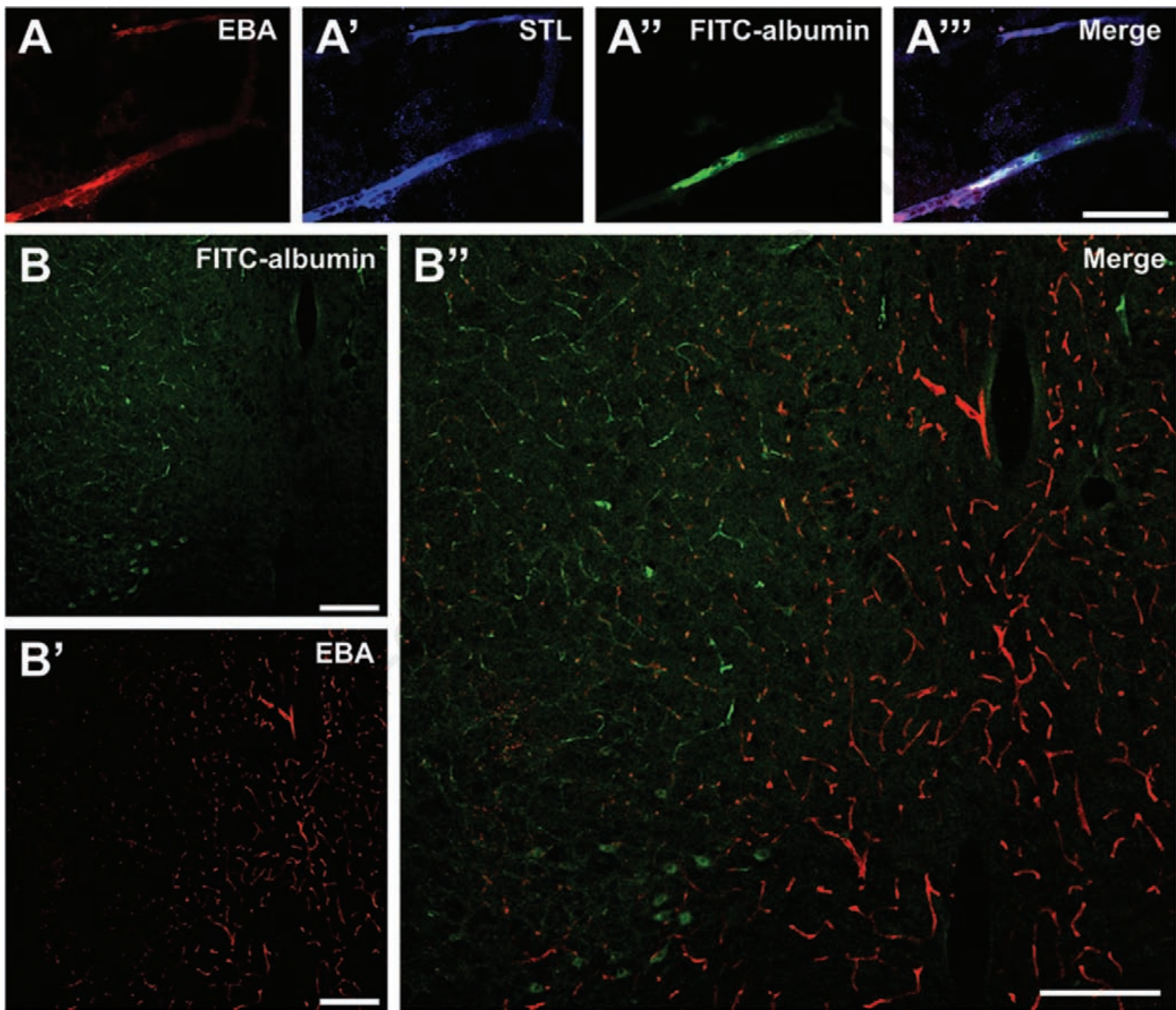

Figure 1. Characteristics of endothelial barrier antigen (EBA)-immunoreactivity associated with ischemia-related decrease in bloodbrain barrier (BBB) integrity, shown in representative coronal rat brain sections within $\mathbf{2 5} \mathrm{h}$ after embolic stroke. Triple fluorescence labeling of EBA (A) and Solanum tuberosum lectin (STL;A') as endothelial markers, as well as fluorescein isothiocyanate FITC-albumin $\left(\mathrm{A}^{\prime \prime}\right)$ as indicator for impaired $\mathrm{BBB}$ integrity revealed a clear allocation in the overlay ( $\left.\mathrm{A}^{\prime \prime}\right)$, validating EBA as part of the vasculature in ischemia-affected brain areas. Detection of extravasated fluorescein FITC-albumin, enhanced by carbocyanine (Cy)2-anti-FITCIgG, marked the area with increased BBB permeability (left part in B). Cy3-immunolabeling of EBA using the SMI-71 antibody resulted in a clearly cut differentiation between the intact appearing vasculature in regions devoid of ischemia (right part in B') and affected vasculature in subcortical ischemic areas (left part in $B^{\prime}$ ), which became obvious by an opposite immunosignal in the merged composition (B"). Scale bars: A,A',A",A", $30 \mu \mathrm{m} ; \mathrm{B}, \mathrm{B}^{\prime}, \mathrm{B}^{\prime \prime}, 200 \mu \mathrm{m}$. 
ischemic conditions. To the best of our knowledge, the present study for the first time provides descriptive details and spatio-temporal characteristics of EBA-immunoreactivity in brain regions with decreased $\mathrm{BBB}$ integrity due to focal cerebral ischemia using an embolic model of stroke in rats.

Five and $25 \mathrm{~h}$ after ischemia induction, both number of EBA-immunopositive vessels and stained area of vessels were reduced in regions with increased BBB permeability. At first glance, these findings confirm previous reports about a decrease of EBA-immunopositive vessels under ischemic conditions, ${ }^{10,11,12}$ but contrary to Lin and Ginsberg ${ }^{10}$ as well as Lu et al. ${ }^{11}$ the stained area per vessel did not change significantly at both time points investigated, probably indicating a lack of vessel fragmentation. This discrepancy might be attributed to several reasons, e.g., differing stroke models (embolic vs filament-based) and localization of measurement (associated with impaired BBB $v s$ infarcted area itself). Indeed, Michalski et al..$^{24}$ could show that the applied model of embolic stroke in rats leads to FITCalbumin leakage that is restricted to ischemiaaffected areas, but vice versa loss of neurons could naturally exceed the area of increased BBB-permeability. Mismatch between previous immunohistochemical studies ${ }^{9-12,28}$ might also result from considerably differing staining methods, e.g., SMI-71 was utilized in dilutions ranging from $1: 100^{28}$ to $1: 2000^{10}$ for immunoperoxidase labeling with diaminobenzidine as chromogen.

So far, a loss of EBA-immunopositive vessels has predominantly been interpreted as a loss of EBA-immunoreactivity linked to an increase in BBB permeability, ${ }_{1}^{13,21,22}$ but specific data on the functional role in focal cerebral ischemia are lacking, especially whether reduced EBAimmunolabeling is the cause or the consequence of BBB dysfunction. Bhattacharjee et al. showed that papaverine, when applied in vivo, resulted in an opening of the $\mathrm{BBB}$ as
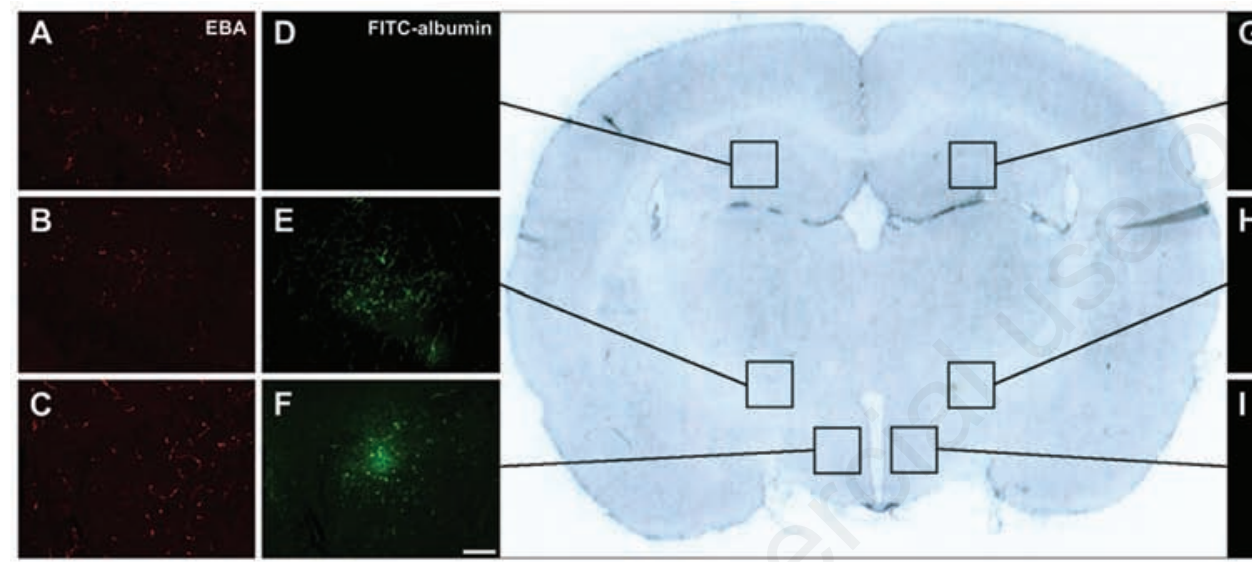

G
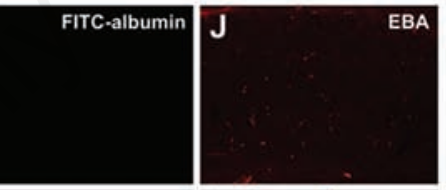

H
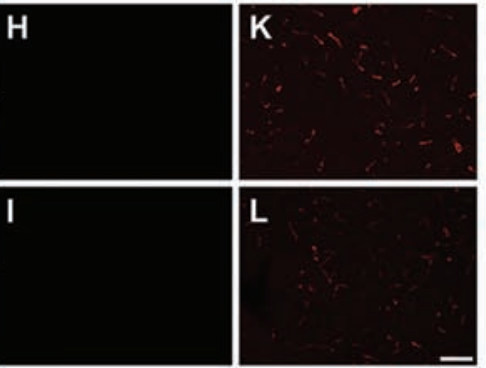

Figure 2. Representative overview scan from a coronal rat brain section $25 \mathrm{~h}$ after embolic stroke (center). Lateral inserts indicate cerebral vessels immunolabeled with anti-endothelial barrier antigen (EBA) on the ischemia-affected hemisphere (A,B,C) and the nonaffected hemisphere (J,K,L), while fluorescein isothiocyanate (FITC)-albumin, enhanced by carbocyanine (Cy)2-anti-FITC-IgG, indicated increased vascular permeability in terms of leakage (D, E and $\mathrm{F}$ on the ischemic $v s \mathrm{G}, \mathrm{H}$ and $\mathrm{I}$ on the contralateral hemisphere). Generally, different parameters of EBA-immunolabeled structures in the striatum (middle inserts) as primarily affected area in the applied model of focal cerebral ischemia, as well as the hypothalamus (lower inserts), which was also involved in several cases, while the hippocampus (upper insets) with apparently intact blood-brain barrier served as control. Scale bars: A-L, $100 \mu \mathrm{m}$.
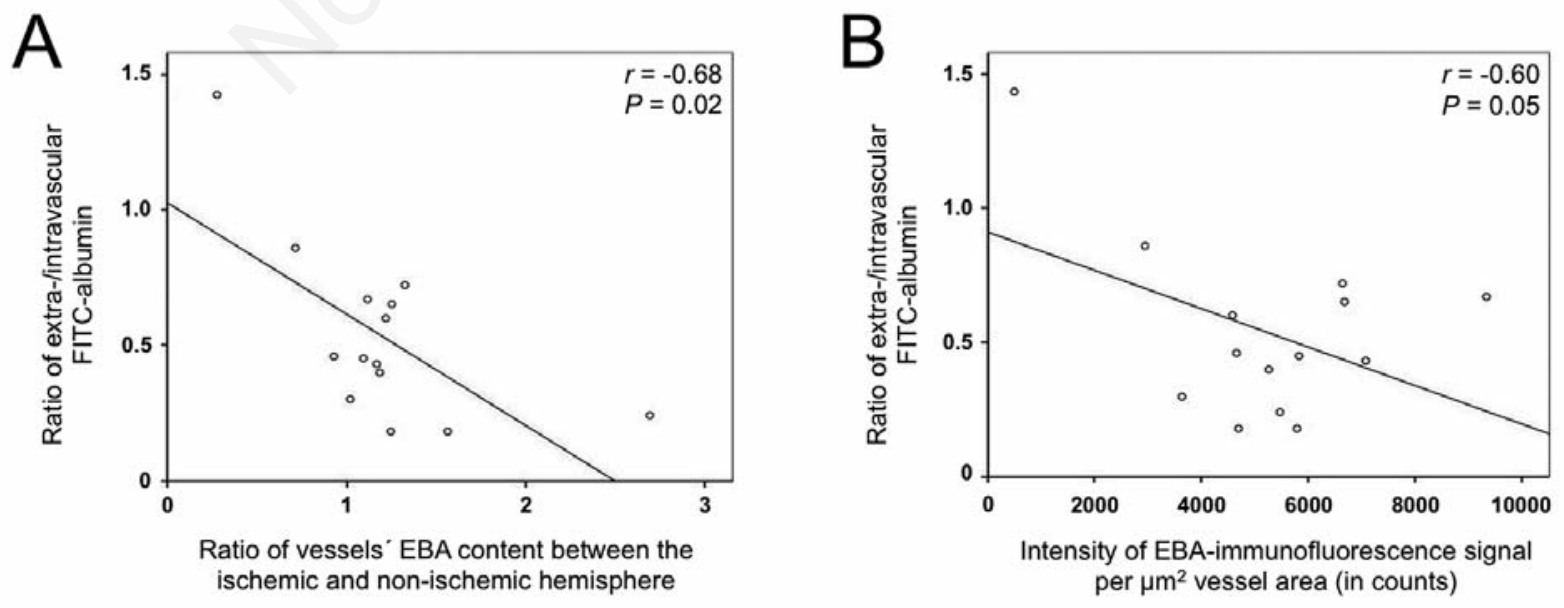

Figure 3. Scatter plots indicating interrelations between the ratio of extra-/intravascular fluorescein isothiocyanate (FITC)-albumin as surrogate for blood-brain barrier integrity and the ratio of the vessels' endothelial barrier antigen (EBA) content between the ischemic and non-ischemic hemisphere (A), as well as the vessels' EBA content (B). 
early as $1 \mathrm{~h}$ after injection, whilst an ongoing reduction of EBA-immunopositive vessels was not seen until $3 \mathrm{~h}$ and an increase of MMP-9 did not occur until $24 \mathrm{~h} .^{28}$ On the other hand, Ghabriel et al. demonstrated that administering anti-EBA in vivo led to a widespread, dosedependent and monophasic opening of the BBB with extravasation of horseradish peroxidase into brain parenchyma. ${ }^{18,29,30}$

In the present study, indirect red fluorescent Cy3-immunolabeling allowed quantitative measurement of vessels' EBA content. Remarkably, as number of EBA-immunopositive vessels and stained vessel area were reduced in regions with FITC-albumin extravasation 5 and $25 \mathrm{~h}$ after ischemia onset, the intensity of the EBA-immunofluorescence signal per $\mu^{2}$ vessel area tended to increase in the remaining vessels. This rise in vessels' EBA content in ischemia-affected areas exhibiting impaired BBB integrity strengthened previous data on a highly dynamic distribution and regulation of EBA, which was also described by Saubaméa et al., even on a cell-tocell basis. ${ }^{20}$ Further support for a highly variable expression level of EBA in endothelial cells emerges from an electron microscopic study showing that EBA was not only located in the luminal membrane of endothelial cells but also in the cytoplasm and nuclei, denoting a pool of EBA which might quickly replenish or reinforce the membranes' content of EBA proteins. ${ }^{31}$ However, even more than 25 years after the first description of anti-EBA recognizing a protein triplet of 30,25 and $23.5 \mathrm{kD}$, respectively, ${ }^{17}$ substantial information on the EBA's protein identity and its exact (patho-)physiological role in the cerebral endothelium is still lacking. With respect to potential effects on BBB integrity, EBA seems to be regulated differently than other well-established BBB markers like the endothelial glucose transporter (GLUT)-1. In a model that used anti-Thy-1 immunotoxin to achieve neuronal loss and astroglial damage, EBA-expression was transiently decreased but nearly completely restored 4 weeks later, while GLUT-1immunoreactivity remained unaltered during the whole observational period. ${ }^{21}$

Furthermore, the total EBA content of vessels in regions with a BBB dysfunction as well as the ratio of the vessels' EBA content between the ischemic and non-ischemic hemisphere were found to correlate significantly with the ratio of extra-/intravascular FITCalbumin as a surrogate for BBB integrity, remarkably, in an inverse manner. To the best of our knowledge, our quantitative data for the first time support the original assumption of Sternberger and Sternberger about EBA's association with parts of the vasculature that possess a selective permeability barrier. ${ }^{17}$ However, the present study revealed no signif- icant correlation between the total number or area of EBA-immunopositive vessels in regions with decreased $B B B$ integrity and the degree of $\mathrm{BBB}$ dysfunction itself.

Concerning the time course of BBB dysfunction after ischemic stroke, currently 2 hypotheses exist: the monophasic model postulates a continuous opening of the BBB beginning as early as 25 min after ischemia induction, ${ }^{32,33}$ while the widely accepted biphasic model describes a first rise in BBB permeability at 3 to $6 \mathrm{~h}$ after ischemia onset, followed by a second opening at 24 to $72 \mathrm{~h}^{34-36}$ Thereby, the biphasic opening is thought to be attributed, amongst others, to a temporally differing release of enzymes degrading components of the NVU, notably MMPs. ${ }^{37,38}$ However, the underlying cause for $\mathrm{BBB}$ stabilization between the first and the second opening is still unclear, ${ }^{8}$ since degrading effects of initially released enzymes would probably continue between these time points. ${ }^{23}$ Considering EBA, the findings of the present study lead to the hypothesis that a pathological stimulus like stroke results in an upregulation of EBA in membranes of still viable cerebral endothelial cells. This increase in vessels' EBA content might be a cause for observed amelioration of BBB dysfunction between the first and the second phase of opening. The transient stabilization of the BBB would also require the involvement of proteins that are not affected by proteases like MMPs. In line with this assumption, no significant correlation was found between the vessels' EBA content and serum levels of MMPs, excluding at least direct effects of MMPs 2 and 9 on EBA.

In summary, our data provide evidence for a functional relationship between EBAimmunoreactivity and BBB dysfunction in a rat model of ischemic stroke, whereas its upregulation is accompanied by an amelioration of BBB dysfunction. Hence, further research is required to clarify the underlying mechanisms of observed dynamics in EBA-immunoreactivity, which might help to identify novel neuroprotective approaches in stroke.

\section{References}

1. O'Collins VE, Macleod MR, Donnan GA, Horky LL, van der Worp BH, Howells DW. 1,026 experimental treatments in acute stroke. Ann Neurol 2006;59:467-77.

2. del Zoppo GJ. Virchow's triad: the vascular basis of cerebral injury. Rev Neurol Dis 2008;5(Suppl. 1):12-21.

3. del Zoppo GJ. The neurovascular unit in the setting of stroke. J Intern Med 2010; 267:156-1.

4. del Zoppo GJ, Hallenbeck JM. Advances in the vascular pathophysiology of ischemic stroke. Thromb Res 2000;98:73-81.

5. Ballabh P, Braun A, Nedergaard M. The blood-brain barrier: an overview. Structure, regulation, and clinical implications. Neurobiol Dis 2004;16:1-13.

6. Bechmann I, Galea I, Perry VH. What is the blood-brain barrier (not)? Trends Immunol 2007;28:5-11.

7. Iadecola C, Nedergaard M. Glial regulation of the cerebral microvasculature. Nat Neurosci 2007;10:1369-76.

8. Sandoval KE, Witt KA. Blood-brain barrier tight junction permeability and ischemic stroke. Neurobiol Dis 2008;32:200-19.

9. Nishigaya K, Yagi S, Sato T, Kanemaru K, Nukui H. Impairment and restoration of the endothelial blood-brain barrier in the rat cerebral infarction model assessed by expression of endothelial barrier antigen immunoreactivity. Acta Neuropathol 2000; 99:231-7.

10. Lin B, Ginsberg MD. Quantitative assessment of the normal cerebral microvasculature by endothelial barrier antigen (EBA) immunohistochemistry:application to focal cerebral ischemia. Brain Res 2000; 865:237-44.

11. Lu A, Clark JF, Broderick JP, PyneGeithman GJ, Wagner KR, Ran R, et al. Reperfusion activates metalloproteinases that contribute to neurovascular injury. Exp Neurol 2008;210:549-59.

12. Suofu Y, Clark J, Broderick J, Wagner KR, Tomsick T, Sa Y, et al. Peroxynitrite decomposition catalyst prevents matrix metalloproteinase activation and neurovascular injury after prolonged cerebral ischemia in rats. J Neurochem 2010;115:1266-76.

13. Katsu M, Niizuma K, Yoshioka H, Okami N, Sakata H, Chan PH. Hemoglobininduced oxidative stress contributes to matrix metalloproteinase activation and blood-brain barrier dysfunction in vivo. $\mathrm{J}$ Cereb Blood Flow Metab 2010;30:1939-50.

14. Sternberger NH, Sternberger LA, Kies MW, Shear CR. Cell surface endothelial proteins altered in experimental allergic encephalomyelitis. J Neuroimmunol 1989; 21:241-8.

15. Lin B, Ginsberg MD, Zhao W, Alonso OF, Belayev L, Busto R. Quantitative analysis of microvascular alterations in traumatic brain injury by endothelial barrier antigen immunohistochemistry. J Neurotrauma 2001;18:389-97.

16. Perdiki M, Farooque M, Holtz A, Li GL, Olsson Y. Expression of endothelial barrier antigen immunoreactivity in blood vessels following compression trauma to rat spinal cord. Temporal evolution and relation to the degree of the impact. Acta Neuropathol 1998;96:8-12. 
17. Sternberger NH, Sternberger LA. Bloodbrain barrier protein recognized by monoclonal antibody. Proc Natl Acad Sci USA 1987;84:8169-73.

18. Ghabriel MN, Lu JJ, Hermanis G, Zhu C, Setchell BP. Expression of a blood-brain barrier-specific antigen in the reproductive tract of the male rat. Reproduction 2002;123:389-97.

19. Cassella JP, Lawrenson JG, Lawrence L, Firth JA. Differential distribution of an endothelial barrier antigen between the pial and cortical microvessels of the rat. Brain Res 1997;744:335-8.

20. Saubaméa B, Cochois-Guégan V, Cisternino S, Scherrmann J. Heterogeneity in the rat brain vasculature revealed by quantitative confocal analysis of endothelial barrier antigen and P-glycoprotein expression. J Cereb Blood Flow Metab 2012;32: 81-92.

21. Krum JM, Kenyon KL, Rosenstein JM. Expression of blood-brain barrier characteristics following neuronal loss and astroglial damage after administration of anti-Thy-1 immunotoxin. Exp Neurol 1997;146:33-45.

22. Zhu C, Ghabriel M, Blumbergs P, Reilly P, Manavis J, Youssef J, et al. Clostridium perfringens prototoxin-induced alteration of endothelial barrier antigen (EBA) immunoreactivity at the blood-brain barrier (BBB). Exp Neurol 2001;169:72-82.

23. Candelario-Jalil E, Yang Y, Rosenberg GA. Diverse roles of matrix metalloproteinases and tissue inhibitors of metalloproteinases in neuroinflammation and cerebral ischemia. Neuroscience 2008;158:983-94.

24. Michalski D, Grosche J, Pelz J, Schneider
D, Weise C, Bauer U, et al. A novel quantification of blood-brain barrier damage and histochemical typing after embolic stroke in rats. Brain Res 2010;1359:186-200.

25. Zhang Z, Zhang RL, Jiang Q, Raman SB, Cantwell L, Chopp M. A new rat model of thrombotic focal cerebral ischemia. J Cereb Blood Flow Metab 1997;17:123-35.

26. Bräuninger S, Kleinschnitz C. Rodent models of focal cerebral ischemia:procedural pitfalls and translational problems. Exp Transl Stroke Med 2009;1:8.

27. Michalski D, Pelz J, Weise C, Kacza J, Boltze J, Grosche J, et al. Early outcome and blood-brain barrier integrity after coadministered thrombolysis and hyperbaric oxygenation in experimental stroke. Exp Transl Stroke Med 2011;3:5.

28. Bhattacharjee AK, Kondoh T, Ikeda M, Kohmura E. MMP-9 and EBA immunoreactivity after papaverine mediated opening of the blood-brain barrier. Neuroreport 2002;13:2217-21.

29. Ghabriel MN, Lu JJ, Tadros R, Hermanis G. A narrow time-window for access to the brain by exogenous protein after immunological targeting of a blood-brain barrier antigen. J Comp Pathol 2004;131:52-60.

30. Ghabriel MN, Zhu C, Hermanis G, Allt G. Immunological targeting of the endothelial barrier antigen (EBA) in vivo leads to opening of the blood-brain barrier. Brain Res 2000;878:127-35.

31. Ghabriel MN, Zhu C, Leigh C. Electron microscope study of blood-brain barrier opening induced by immunological targeting of the endothelial barrier antigen. Brain Res 2002;934:140-51.

32. Strbian D, Durukan A, Pitkonen M,
Marinkovic I, Tatlisumak E, Pedrono E. The blood-brain barrier is continuously open for several weeks following transient focal cerebral ischemia. Neuroscience 2008;153:175-81.

33. Durukan A, Marinkovic I, Strbian D, Pitkonen M, Pedrono E, Soinne L. Postischemic blood-brain barrier leakage in rats:One-week follow-up by MRI. Brain Res 2009;1280:158-65.

34. Preston E, Sutherland G, Finsten A. Three openings of the blood-brain barrier produced by forebrain ischemia in the rat. Neurosci Lett 1993;149:75-8.

35. Belayev L, Busto R, Zhao W, Ginsberg MD. Quantitative evaluation of blood-brain barrier permeability following middle cerebral artery occlusion in rats. Brain Res 1996; 739:88-96.

36. Pillai DR, Dittmar MS, Baldaranov D, Heidemann RM, Henning EC, Schuierer G, et al. Cerebral ischemia-reperfusion injury in rats - A 3 T MRI study on biphasic bloodbrain barrier opening and the dynamics of edema formation. J Cereb Blood Flow Metab 2009;29:1846-55.

37. Rosenberg GA, Estrada EY, Dencoff JE. Matrix metalloproteinases and TIMPs are associated with blood-brain barrier opening after reperfusion in rat brain. Stroke 1998;29:2189-95.

38. Yang Y, Estrada EY, Thompson JF, Liu W, Rosenberg GA. Matrix metalloproteinasemediated disruption of tight junction proteins in cerebral vessels is reversed by synthetic matrix metalloproteinase inhibitor in focal ischemia in rat. $\mathrm{J}$ Cereb Blood Flow Metab 2007;27:697-709. 